\title{
Care Outcomes for Chiropractic Outpatient Veterans (COCOV): a qualitative study with veteran stakeholders from a pilot trial of multimodal chiropractic care
}

\author{
Stacie A. Salsbury ${ }^{1 *}\left(\mathbb{D}\right.$, Elissa Twist $^{1}$, Robert B. Wallace ${ }^{2}$, Robert D. Vining ${ }^{1}$, Christine M. Goertz ${ }^{3}$ and \\ Cynthia R. Long ${ }^{1}$
}

\begin{abstract}
Background: Low back pain (LBP) is common among military veterans seeking treatment in Department of Veterans Affairs (VA) healthcare facilities. As chiropractic services within VA expand, well-designed pragmatic trials and implementation studies are needed to assess clinical effectiveness and program uptake. This study evaluated veteran stakeholder perceptions of the feasibility and acceptability of care delivery and research processes in a pilot trial of multimodal chiropractic care for chronic LBP.

Methods: The qualitative study was completed within a mixed-method, single-arm, pragmatic, pilot clinical trial of chiropractic care for LBP conducted in VA chiropractic clinics. Study coordinators completed semi-structured, in person or telephone interviews with veterans near the end of the 10-week trial. Interviews were audiorecorded and transcribed verbatim. Qualitative content analysis using a directed approach explored salient themes related to trial implementation and delivery of chiropractic services.
\end{abstract}

Results: Of 40 participants, 24 completed interviews (60\% response; $67 \%$ male gender; mean age 51.7 years). Overall, participants considered the trial protocol and procedures feasible and reported that the chiropractic care and recruitment methods were acceptable. Findings were organized into 4 domains, 10 themes, and 21 subthemes. Chiropractic service delivery domain encompassed 3 themes/8 subthemes: scheduling process (limited clinic hours, scheduling future appointments, attendance barriers); treatment frequency (treatment sufficient for LBP complaint, more/less frequent treatments); and chiropractic clinic considerations (hire more chiropractors, including female chiropractors; chiropractic clinic environment; patient-centered treatment visits). Outcome measures domain comprised 3 themes/4 subthemes: questionnaire burden (low burden vs. time-consuming or repetitive); relevance (items relevant for LBP study); and timing and individualization of measures (questionnaire timing relative to symptoms, personalized approach to outcomes measures). The online data collection domain included 2 themes/4 subthemes: user concerns (little difficulty vs. form challenges, required computer skills); and technology issues (computer/internet access, junk mail). Clinical trial planning domain included 2 themes/5 subthemes: participant recruitment (altruistic service by

\footnotetext{
*Correspondence: stacie.salsbury@palmer.edu

1 Palmer Center for Chiropractic Research, Palmer College of Chiropractic,

741 Brady Street, Davenport, lowa 52803, USA
}

Full list of author information is available at the end of the article

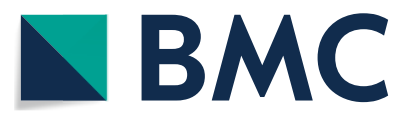

(c) The Author(s) 2022. Open Access This article is licensed under a Creative Commons Attribution 4.0 International License, which permits use, sharing, adaptation, distribution and reproduction in any medium or format, as long as you give appropriate credit to the original author(s) and the source, provide a link to the Creative Commons licence, and indicate if changes were made. The images or other third party material in this article are included in the article's Creative Commons licence, unless indicated otherwise in a credit line to the material. If material is not included in the article's Creative Commons licence and your intended use is not permitted by statutory regulation or exceeds the permitted use, you will need to obtain permission directly from the copyright holder. To view a copy of this licence, visit http://creativecommons.org/licenses/by/4.0/. The Creative Commons Public Domain Dedication waiver (http://creativeco mmons.org/publicdomain/zero/1.0/) applies to the data made available in this article, unless otherwise stated in a credit line to the data. 
veterans, awareness of chiropractic availability, financial compensation); and communication methods (preferences, potential barriers).

Conclusions: This qualitative study highlighted veteran stakeholders' perceptions of VA-based chiropractic services and offered important suggestions for conducting a full-scale, veteran-focused, randomized trial of multimodal chiropractic care for chronic LBP in this clinical setting.

Trial registration: ClinicalTrials.gov NCT03254719

Keywords: Veterans health, Stakeholder participation, Qualitative research, Health services, Health communication, Chiropractic, Low back pain, Data collection, Patient-reported outcome measures, Pilot study

\section{Key messages regarding feasibility}

- What uncertainties existed regarding feasibility?

While chiropractic services are provided within VA healthcare facilities, the acceptability and feasibility of conducting pragmatic randomized trials of multimodal chiropractic care for low back pain in this setting was unknown.

- What are the key feasibility findings?

Qualitative interviews were conducted with the veterans who enrolled in this pilot trial to evaluate the acceptability of research methods and clinical care delivery. Veterans make key recommendations about treatment scheduling, reasons for participant involvement, and patient communication needs. Interviews clarified the burden and relevance of outcome measures and ways to better facilitate online data collection. Veterans also provided insights into the delivery of chiropractic treatments for this patient population.

- What are the implications of the feasibility findings for the design of the main study?

Findings suggest pragmatic trials of chiropractic care are feasible in VA settings, and that the participant recruitment strategies, treatment scheduling processes, selected outcome measures, and online data collection procedures used in this pilot were acceptable to most veterans. Compensation for completion of qualitative interviews was added to the study protocol to improve participation in this important component of the main study.

\section{Background}

Military veterans often wage long-term battles with the overlapping problems of chronic pain and mental illness following discharge from the armed services [1]. More than $50 \%$ of U.S. veterans experience chronic pain syndromes, with pain intensity rated as moderate to severe [2]. Chronic pain prevalence rates increase each year following deployment [3] and may be more prevalent in female veterans [4]. Musculoskeletal pain is especially common, with chronic low back pain (LBP) impacting $25 \%$ of veterans [1]. Concurrent with musculoskeletal pain, many veterans also report high rates of depression, anxiety, post-traumatic stress disorder (PTSD), and substance use disorder, among other mental health comorbidities [1, 5]. Veterans with co-occurring chronic musculoskeletal pain and mental health conditions have higher rates of healthcare service utilization and greater use of prescription psychotropic and pain medication, including opioids $[5,6]$.

In response, the U.S. Department of Veterans Affairs, Veterans Health Administration (VA) has instituted policy efforts to improve clinical pain management and combat the opioid epidemic. One prominent recommendation is to integrate non-pharmacological and complementary and integrative health $(\mathrm{CIH})$ approaches into VA primary care, pain care, and mental health settings when sufficient evidence of safety and effectiveness exists [7-9]. Nine approaches have been prioritized for early integration, including psychological and behavioral therapies; exercise and movement therapies; manual therapies including spine and joint manipulation; and team-based, multimodal pain care [8]. Many veterans with pain-related conditions use these, and other, $\mathrm{CIH}$ approaches regularly, with utilization rates trending upwards and ranging from $1-52 \%$ by therapy and study [9-12].

Sufficient evidence currently exists on the safety and effectiveness of spinal manipulation, a central component of multimodal chiropractic care, to recommend this treatment for veterans with LBP and neck pain [13]. In addition, veterans who receive chiropractic care may be less likely to use opioid medications [14]. Currently, however, few prospective studies on chiropractic care for veterans are available on which to base study protocols for randomized controlled trials (RCTs) designed to determine dosage and treatment parameters $[15,16]$. 
Veterans increasingly choose chiropractic care as a therapeutic option, with both the number of patients served and treatment visits completed annually rising steadily since services were introduced into VA healthcare facilities in 2004 [17, 18]. In tandem with efforts to improve pain management services, VA has proposed research agendas for non-pharmacological treatments for chronic musculoskeletal pain [13]. Crucial scientific priorities remain for chiropractic, including implementation research to facilitate clinical adoption and patient access; effectiveness trials for non-spine-related musculoskeletal conditions; and research conducted with underrepresented veteran populations, including older adults, women, and patients with comorbidities, such as mental health concerns [13].

Toward this agenda, our team conducted a mixedmethod, single-arm, pragmatic, pilot clinical trial of multimodal chiropractic care for veterans with chronic LBP and with or without mental health comorbidity. The aim of the Care Outcomes for Chiropractic Outpatient Veterans (COCOV) pilot trial was to evaluate the feasibility, safety, and acceptability of multimodal chiropractic care for a veteran population. The objectives of this qualitative study were to (1) identify veteran perceptions of the acceptability and feasibility of the trial's research processes and (2) report participant recommendations for chiropractic services in VA settings.

\section{Methods \\ Design}

The research design was a qualitative interview study nested within a single-arm, pilot clinical trial of multimodal chiropractic care for veterans with chronic LBP [19]. This qualitative study used a descriptive, phenomenological perspective to understand veterans' experiences with the research methods used in this pilot study and their suggestions for chiropractic services offered within VA settings [20,21]. Additional file 1 provides the Consolidated Criteria for Reporting Qualitative Studies (COREQ) checklist for this study [22].

\section{Clinical trial background}

Clinical trial design and primary results are presented in a companion article (Long CR, Salsbury SA, Vining RD, Lisi AJ, Corber L, Twist EJ, Abrams T, Wallace RB, Goertz CM: Care Outcomes for ChiropraLong CR, Salsbury SA, Vining RD, Lisi AJ, Corber L, Twist EJ, Abrams T, Wallace RB, Goertz CM: Care Outcomes for Chiropractic Outpatient Veterans (COCOV): a single-arm, pragmatic, pilot trial of multimodal chiropractic care for U.S. veterans with chronic low back pain, Submitted). Briefly, eligible veterans received up to 10 weeks of chiropractic care to address chronic LBP and back-related disability. Participants received on average 4.5 treatment visits (described further in results). Multimodal chiropractic care consisted of spinal manipulative therapy, spinal mobilization, other manual therapies, active exercise, and lifestyle advice delivered by licensed doctors of chiropractic (DC). An integrative care pathway for veterans with LBP also was provided as a reference for treatment decisions and referrals to primary care and mental health providers [19]. Outcome measures were completed online via REDCap (Research Electronic Data Capture, Vanderbilt, Nashville, TN, USA) at baseline and weeks 3, 5, 7, and 10 [23]. Outcome measures included recommended biomedical and psychosocial parameters [24], with the Roland Morris Disability Questionnaire as the primary outcome [25]. Patient-reported outcomes included established questionnaires and select instruments from the National Institutes of Health (NIH) Patient-Reported Outcome Measurements Information System (PROMIS ${ }^{\circledR}$ ) [26] and the Pain Assessment Screening Tool and Outcomes Registry (PASTOR) [27], which were piloted with the legacy measures to evaluate their usefulness in veteran populations.

\section{Setting}

The setting was a 2-site chiropractic clinic located in the State of Iowa, within Veterans Integrated Service Network (VISN) 23 of the VA Midwest Health Care Network. VISN-23 serves more than 440,000 veterans in parts of 11 states in the midwestern United States, including Iowa. Chiropractic services were provided through the Chiropractic/Acupuncture Clinic in the Extended Care and Rehabilitation Service Line. At the time of this trial, one chiropractic clinic was co-located with a pain clinic at the Iowa City VA Health Care System in Iowa City, Iowa, while the second was co-located within primary care at a community-based outpatient clinic in Coralville, Iowa. Two licensed DCs employed by VA provided chiropractic care. Although acupuncture was available at these chiropractic clinics, this modality was not delivered in the trial. Veterans received usual medical and mental health services from their current providers, with a chiropractic integrated care pathway provided as a resource for clinical evaluation and interprofessional communication and referrals [19].

\section{Participants}

Eligibility criteria for trial participation are described in a companion article (Long CR, Salsbury SA, Vining RD, Lisi AJ, Corber L, Twist EJ, Abrams T, Wallace RB, Goertz CM: Care Outcomes for ChiropraLong CR, Salsbury SA, Vining RD, Lisi AJ, Corber L, Twist EJ, Abrams T, Wallace RB, Goertz CM: Care Outcomes for Chiropractic Outpatient Veterans (COCOV): a single-arm, pragmatic, 
pilot trial of multimodal chiropractic care for U.S. veterans with chronic low back pain, Submitted). Briefly, veterans age 18 years and older who reported chronic LBP consistent with the NIH Task Force on Research Standards for Chronic Low Back Pain [24] definition (LBP of at least 3 months duration and pain on at least half the days in the past 6 months) were eligible for the trial. Potential interview subjects included all trial participants, with no exclusions to the interview. Our recruitment goal was 40 participants, the sample size chosen to adequately assess the feasibility of participant recruitment and retention strategies, as well as electronic data collection processes. The sample was to include at least $20 \%$ female participants in both the trial and interviews, as oversampling of women veterans is a recommendation from VA women's health research experts [28]. Participant recruitment for the clinical trial (also described in the companion article) included provider referrals, chiropractic clinic patient screening, focused mailings based on key characteristics identified in the electronic health record, and standard direct recruitment with posters placed in VA health settings and veteran-centric organizations. Study coordinators telephoned trial participants to invite them to complete the qualitative interview.

\section{Data collection}

Interviews were scheduled from 2 weeks before until 2 months after a participant completed the 10-week trial. Two study coordinators conducted the interviews, either in person at the VA chiropractic clinics or by telephone in a call originating from the Palmer Center for Chiropractic Research in Davenport, IA. Participants read information about the interview during the informed consent process and received a verbal overview of its procedures before the interview. A structured question list (Table 1) guided the interview, with topics focused on participant perspectives of the chiropractic intervention and service delivery [29] and the feasibility and acceptability of the clinical trial procedures, as guided by the $\mathrm{CON}$ SORT checklist [30]. Participants were encouraged to elaborate on aspects of the trial they found difficult or challenging to complete through probes on more closedended questions or when brief replies were offered. For example, follow-up questions asked participants about outcomes that were important to them, but which were not included on data collection forms. Interviews were recorded using digital recording devices and uploaded to a secure website for professional transcription (Way With Words, New York). Transcripts were reviewed against the audiorecordings to assess accuracy by the lead qualitative investigator.

\section{Data analysis}

This qualitative content analysis used a directed approach to identify salient topics for the implementation of a full-scale randomized clinical trial of chiropractic care within VA [31], such as treatment scheduling, communication processes, outcome measures, data collection procedures, and chiropractic clinic recommendations [32]. The primary data analyst (SAS) achieved familiarity with the text through open reading of the complete transcripts, with successive transcript readings identifying discrete topics of interest aligning with key issues in clinical trial implementation. Meaning units were identified, with salient passages from the transcript transferred to a spreadsheet by participant identification number (PTID) and coding domain. Subsequent analysis rounds were organized into data tables to form patterns that included sub-coding within each domain to categorize emerging themes and subthemes. Coding continued until no new themes were identified. Data tables were provided to coinvestigators and study staff for comment, clarification, and revision (see Additional files 1 and 2). Representative quotes were offered by PTID with names and/or gender removed whenever possible to allow anonymity of study participants and DCs.

Table 1 Interview questions

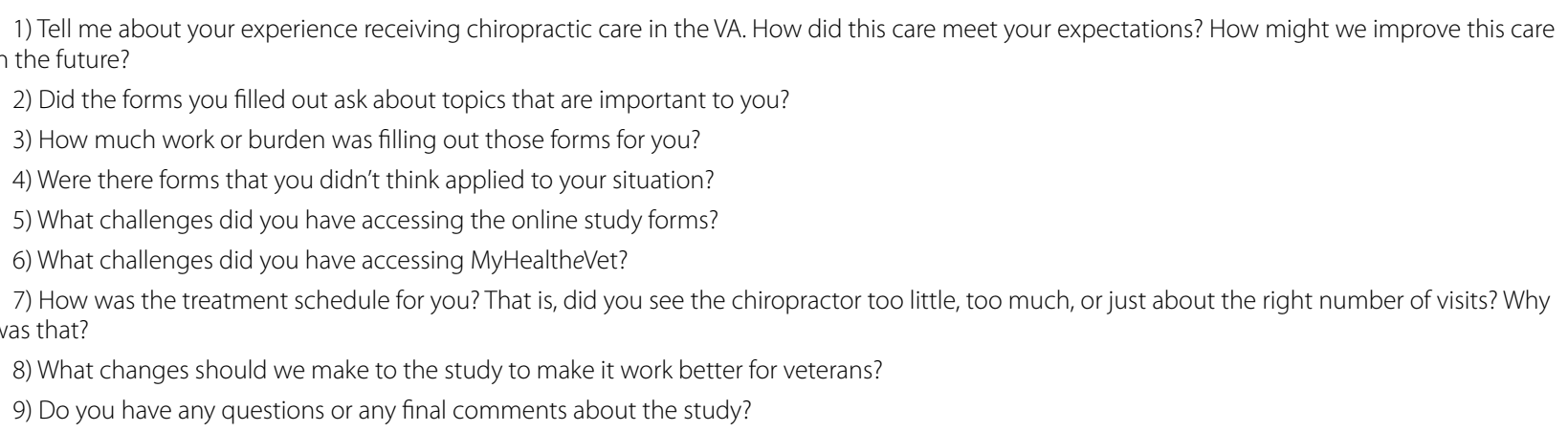




\section{Ethical considerations}

Ethics approvals were granted by the Palmer College Foundation Institutional Review Board (IRB), The University of Iowa IRB (IRB-03 VA Only), and the VA Connecticut Health System IRB. Participants provided written informed consent to enroll in the trial which included information about the qualitative component of the research. Veterans completed VA Authorization Form \#10-0493 to allow the creation and use of an audiorecording for research purposes. Participants also gave verbal consent to the recording process before the interview. Participants received up to $\$ 100$ in gift cards for completion of primary outcome measures collected via REDCap during the trial, although veterans received no additional incentive to complete the qualitative interview.

\section{Results}

\section{Characteristics of the sample and interviews}

Of 40 trial participants, 24 completed interviews $(60 \%$ response). Male veterans $(n=16 ; 67 \%)$ predominated the interviews, although the inclusion of 8 women veterans (33\%) as interview participants achieved our goal of a minimum $20 \%$ female sample. Mean age (SD) was 51.7 (15.7) years with most participants stating their race as white (88\%) and their ethnicity as non-Hispanic or Latinx (96\%). All participants (100\%) stated chronic LBP was an ongoing problem for more than 6 months. As consistent with our eligibility criteria, which included veterans with or without selected mental health conditions, most participants (95\%) had either a history of mental health comorbidity documented in their electronic health record or positively screened for depression, anxiety and/or post-traumatic stress disorder on their baseline outcomes.

Interview duration averaged 16:46 (range 6:42 to 42:37), with 11 conducted face-to-face at the VA chiropractic clinics and 13 completed as telephone interviews. Of the 16 participants who did not complete an exit interview, 5 were not able to be contacted by study staff while the remainder had scheduling conflicts, or the veteran decided to not participate.

\section{Overall findings}

Study findings (Fig. 1) provided critical information for the planning of a full-scale, pragmatic randomized clinical trial of chiropractic care in VA settings that was subsequently funded by NIH [31]. Our results also offer a view into the patient perspective of the delivery of chiropractic care within VA. Domain 1 related to chiropractic service delivery in VA, emphasizing processes related to scheduling and attending chiropractic treatments. Domains 2 and 3 addressed methodological concerns for conducting

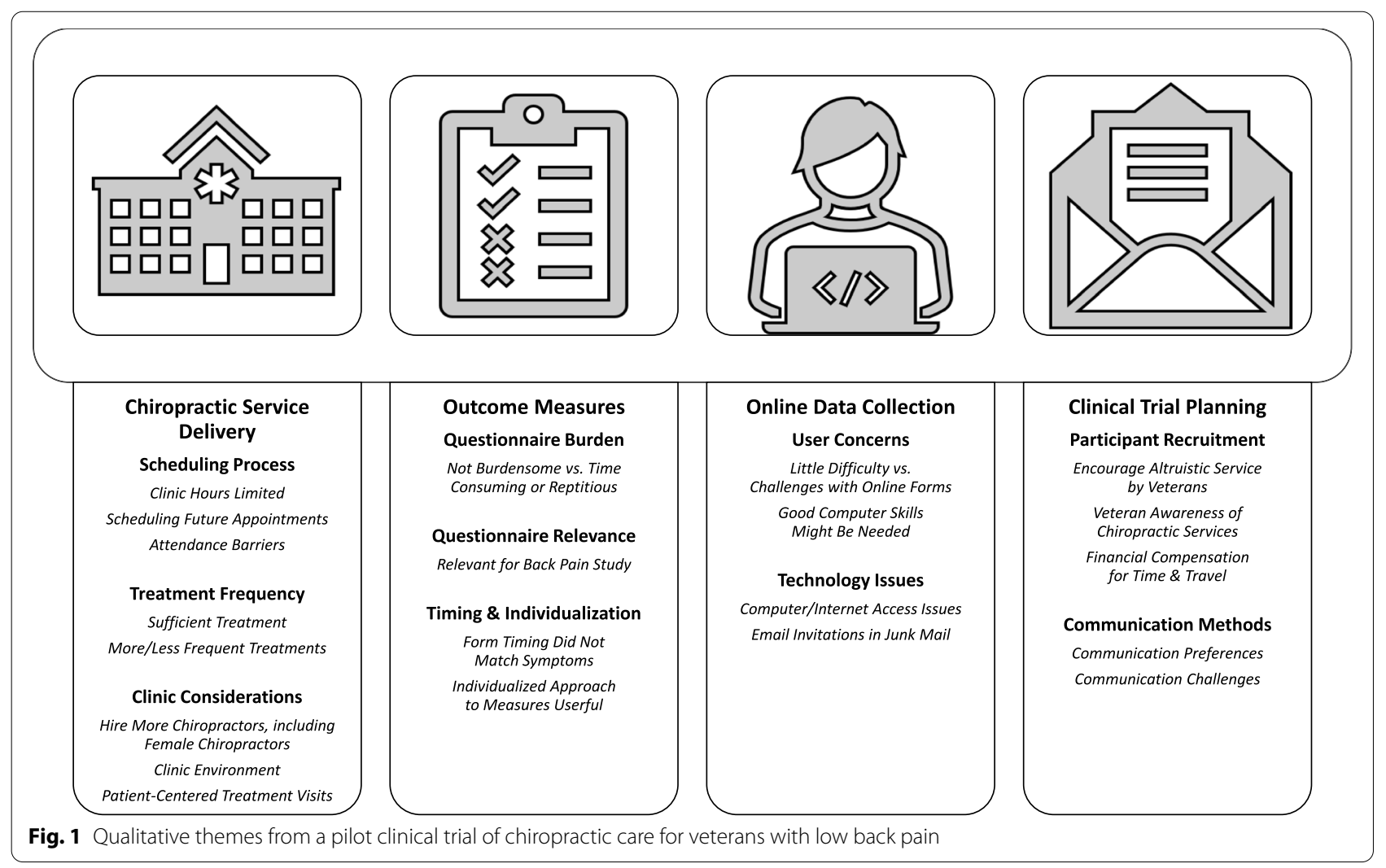


chiropractic clinical trials within VA, highlighting patient perceptions of selected outcome measures and the logistics of the online data collection. Domain 4 offers insights into other clinical trial planning considerations, including participant recruitment and communication.

\section{Domain 1: Chiropractic service delivery for a clinical trial in VA}

Domain 1 outlined veterans' perspectives about the delivery of chiropractic services within a clinical trial conducted in VA settings. Themes included the scheduling process, treatment frequency, and patient-centered clinic environments, including personnel staffing.

\section{Theme 1: Scheduling process for chiropractic services}

Participants offered positive $(n=10)$ or mixed $(n=9)$ comments about their scheduling experiences, with DCs and clinic staff helping navigate the process. Negative comments $(n=5)$ pertained to unexpected delays in appointments, with some reporting waits of 2 weeks to 1 month.

Clinic hours limited Veterans noted the VA clinic offered limited hours (8:00 a.m.-4:30 p.m.) and weekday-only appointments, which some viewed negatively against the ease with which chiropractic care could be scheduled in community settings.

Different hours ... Some of us can't get down here at two o'clock because we work, but I would not stop this ... Wouldn't change a thing except for the times. (PTID:88)

[VA clinic] only takes [patients] until 3:30pm, where I could go to an outside chiropractor at $6 \mathrm{pm}$ at night, or on a Saturday morning. (PT:167)

Scheduling out Several veterans reported "scheduling out" DC appointments, even if they were not experiencing symptoms. Such appointments were made before activities that might trigger LBP, such as work rotations or training events, and canceled if not needed.

I know it's extremely difficult to get in and make appointments. I like to try and make appointments one full month out because otherwise, they'd fill up quickly. (PTID:4)

Attendance barriers Additional attendance barriers included the travel distance to the VA chiropractic clinic, vacations, and other health problems.

I see the chiropractor when I can get in ... based on my travel problems that I've got... Since I bounce back and forth between [two states], it's sometimes difficult to make an appointment. (PTID:82)

\section{Theme 2: Treatment frequency during clinical trial}

Most participants were scheduled to receive chiropractic care once weekly, with treatment frequency determined by clinical need and patient scheduling preferences. During the trial, participants had mean attendance of 4.5 visits (range 1 to 7 ) over 10 weeks. Many participants reported this treatment frequency, or dosage, of chiropractic care was sufficient to address their LBP complaint, although some veterans recommended more or less frequently scheduled treatments.

Treatment frequency sufficient for LBP complaint Many participants reported the weekly treatments received in this study were sufficient to address their primary clinical concern.

Just right. We had good communication and we talked about how I felt. The amount of visits I needed to be seen for versus how much [the chiropractor] thought. We discussed together and made that treatment plan. (PTID:135)

More or less frequent treatments desired Some participants thought frequent treatments, scheduled earlier in the trial, might be beneficial while others wanted less frequent visits.

When we started and [DC] did the first one [treatment], it was great. But then it was a long period before I got to see $[\mathrm{DC}]$ the second time. I think more frequently at the beginning of the pain is better than waiting a month later because it almost puts you back where you were. (PTID:88)

I saw [DC] every 2 weeks. I thought it was excessive, but [DC] thought that was what was necessary and [the DC] is the doctor, so I take that advice. (PTID-52)

\section{Theme 3: VA chiropractic clinic considerations}

Veterans offered many recommendations to impact VA chiropractic clinics, such as hiring additional DCs including female chiropractors, improving the clinic environment, and creating patient-centered treatment visits.

Additional VA chiropractors on staff, including female DCs Several participants recommended VA hire more DCs to improve service delivery within the healthcare 
system, including female chiropractors for veterans with this preference.

Maybe getting more available? About 2 to 3 weeks before I can get back in with [the chiropractor]. There's a lot of other people that need to see [chiropractor]. (PTID: 94)

What about female staff? Because a patient like me that has MST [military sexual trauma] and PTSD, I can only work with female providers. There's a lot of individual needs but if a need is a female provider, make sure that one is available. Make sure you have a balance between male and female participants and male and female care providers. That's really important ... ... you don't want to cause more stress. (PTID:187)

Chiropractic clinic environment Some improvements to the chiropractic clinic environment were suggested, including lighting, room layout, and a more 'healing' atmosphere.

It's the VA. What that means is it's more of a hospital setting ... I don't necessarily get that place-of-healing-at-theVA feeling. Construction projects in the hallway. Random people yelling outside. The room was a little small ... huge halogen lights in the office. That bright light, when you're laying down, if you have a migraine, it's really hard to deal with. (PTID: 15)

Patient-centered treatment visits Veterans suggested ways to make treatment visits more patient-centered, including providing patient education materials about chiropractic, LBP diagnoses, and self-care strategies, as well as demonstrating how chiropractic equipment works before the manipulation, especially with patients who have anxiety or PTSD.

Me, being who I am, and in the state I am in with PTSD, [treatment] was a lot to get used to, especially using the machine. It makes kind of a weird noise ... the only thing I would recommend different is let me hear that gun [chiropractic instrument] before it's used on my body when I'm lying face down with my eyes closed. That freaked me out. (PTID: 6)

\section{Domain 2: Selected outcome measures}

Descriptive statistics reporting completion times and rates for the outcome measures are reported in the companion article (Long CR, Salsbury SA, Vining RD, Lisi AJ, Corber L, Twist EJ, Abrams T, Wallace RB, Goertz CM: Care Outcomes for Chiropractic Outpatient Veterans
(COCOV): a single-arm, pragmatic, pilot trial of multimodal chiropractic care for U.S. veterans with chronic low back pain, Submitted). During interviews, participants reflected upon the content and experience of completing outcome measures (referred to as study forms) during the pilot. Themes discussed questionnaire burden, questionnaire relevance, and the timing and individualization of outcome measures.

\section{Theme 1: Questionnaire burden}

Burden Many veterans $(n=22)$ stated the study forms were easy to follow and not considered burdensome to complete.

As far as filling out the questionnaires, they were easy. They did not take as long as I thought any of them would take ... very minimal burden. (PTID:52)

Time-consuming or repetitious Some individuals said completing the questionnaires was time-consuming, while others commented on the repetitious nature of some measures.

Well, not so much as a burden as much as time consuming and a lot of repetitive questions, just asked a different way or under a different heading I guess. (PTID: 94)

\section{Theme 2: Questionnaire relevance}

Relevance Most participants agreed the outcome measures were relevant to their experience of LBP. However, some veterans questioned the rationale for including questions about mental health, substance abuse, suicide, or one's outlook on life.

PTSD and depression ... is a huge thing with veterans. But really, I'm going to say it. It gets old, answering the same question every time I go somewhere. 'Do you feel like killing yourself?' Well no, I don't feel like killing myself ... I can't go anywhere in the VA system or take any study ... without someone asking me if I feel like killing myself. (PTID:114)

The only one that I was a little bit miffed by or quizzical about was the one about religion. I didn't know how that fit with back pain. Do I believe in this, that and the other, and I'm going, 'What does this have to do with my back pain?' (PTID:85) 


\section{Theme 3: Questionnaire timing and individualization}

Timing Several participants reported that the timing of the outcome measures did not coincide with the presentation or fluctuations in their symptoms:

Answering the questions, sometimes it's difficult because on that particular day or even that week, my pain might have been a 5 or a 6 where maybe the following week, I didn't even have a $1 . .$. it's not a constant thing with me ... it's just hard to put it in a box. (PTID: 111)

Individualized approaches Several people endorsed questionnaires that allow for flexibility or individualization, such as items about activities important to the patient. However, some stated the narrow focus on LBP prevented full evaluation of associated spine-related conditions, such as neck pain. Some suggested adding open field text boxes to allow participants to make personalized comments about their health or study experience.

It asked the 3 things that are important to me. There are some things I want to be able to do that I can't really do or have a hard time doing ... spending time with my 7 year-old son ... working on the farm ... you've got to have all that information to get the proper care and treatment. (PTID: 187)

I was having a lot of problems with my neck and there wasn't very many questions asking about my neck ... If I had a place to make a comment of my own, then I could have told you about my neck. There is no place for me to do that. (PTID: 186)

\section{Domain 3: Online data collection procedures}

Trial data collection procedures highlighted user concerns and technology issues. Most veterans completed all study-related forms online using REDCap without apparent difficulty, although some completed abbreviated outcome measures via a computer-assisted telephone interview. Two veterans mentioned a preference for completing paper forms.

It would be kind of cool if you went and did your therapy, and maybe ... I don't know. If I could have requested it [the questionnaires] on paper. (PTID:4)

\section{Theme 1: User concerns}

Many participants $(n=13)$ reported little difficulty completing questionnaires using REDCap, noting the email notifications, ease with which surveys could be restarted, and the convenience of using smartphones to complete questionnaires.

It wasn't difficult. I think every single one I did on my phone ... click the button. Take the survey. (PTID:114)

Challenges completing online forms Some participants reported being "locked out" when they missed a data collection window. Others were unsure of questionnaire timing (how often the forms would be completed) or duration (how long each form would take to complete).

I thought I started off strong and yet I did not complete ... the online questionnaires on time and therefore I was locked out of those. I feel badly about not completing that part of the survey. (PTID:4)

Good computer skills needed While many participants noted their own "computer savviness," some wondered how older veterans or persons with low technology skills might fair.

Someone, if they were not really good with technology, they might struggle with it a bit, but I didn't say it was a problem. (PTID:6)

\section{Theme 2: Technology issues}

Computer or internet access Eleven participants reported issues completing online forms due to limited internet access in rural locations or personal decisions about computer technology.

Well, I live in the country so sometimes the internet's sketchy. Sometimes it works, sometimes it doesn't. (PTID:6)

It's not that it was difficult for me. I just don't have a computer at home. I don't have Wi-Fi. I don't have internet cable. Where I work, I do. I did my surveys when I was at work. But you know? If I didn't have that option, I probably wouldn't have been able to do the surveys at all. (PTID:150)

Email invitations going to junk mail Participants received training on configuring their email to accept REDCap messages, but several reported these emails went to their "junk mail" folders.

I don't know how you prevent this. I have to go into my junk folder and look for them. Otherwise, I miss them. [Study staff] sent me a text message ... reminder ... and 
there was actually an email and a reminder in my junk folder. (PTID:114)

\section{Domain 4: Clinical Trial Planning Considerations}

Participants described other experiences in this pilot that influenced planning for the full-scale clinical trial. Veterans endorsed established recruitment strategies, such as brochures and having providers introduce patients to studies, and recommended encouraging altruistic service by veterans, increasing veteran awareness about VA chiropractic services, and offering financial compensation. Methods to contact veterans focused on preferences and challenges.

\section{Theme 1: Participant recruitment}

Encourage altruism Several participants considered their participation in the trial as an opportunity to serve other veterans, especially those with back pain.

I received a call and I figured I could be of use. I was recently out of the service for back problems. (PTID: 162)

Awareness of chiropractic services Some veterans reported not knowing much about the health profession of chiropractic. Others did not know VA offered chiropractic care.

Why I did this study was because I was unaware I could get chiropractic care at the VA. (PTID:150)

The chiropractic. It's just I haven't had that experience before. (PTID:137)

Financial compensation Some participants asked how study involvement would be billed or impact travel reimbursement; several appreciated the financial compensation for their time.

[Gift card] is going to buy my hunting stuff ... that's a benefit right there. I mean, that makes you want to do the study. (PTID:167)

\section{Theme 2: Communication methods}

Communication preferences Participants' preferred methods of communication with study personnel included telephone (45\%) or text message (55\%). Most participants reported no communication problems, although 2 reported challenges with contacts by telephone.

I got all of the emails or answering machine, but [study staff] never answers me back ... we just couldn't get together on a time. Wasn't your fault, wasn't my fault. It just came together today. (PTID:186)

Veterans were asked about MyHealtheVet (MHV), the VA web-based, personal health record, as a communication tool. Twelve used MHV regularly for visit reminders, provider contacts, record access, and prescription refills. Non-users $(n=7)$ or those unsure if they used MHV ( $n$ $=4$ ) reported difficulties with internet connection or application functions; password changes; and preferences for receiving appointment reminders by phone.

I signed up because I wanted to get text messages ... now I get my appointments texted to me. (PTID:15)

Communication barriers Some veterans reported concurrent enrollment in other VA studies, which was a challenge during interviews or when filling out surveys. A few veterans reported memory, mental health concerns, or other health issues which made communication difficult.

They need to follow up a little bit more, because us veterans are forgetful. I have combat PTSD. I take a lot of meds. And I am forgetful ... [staff] need to follow up more with the patient, to get him an appointment. (PTID:167)

\section{Discussion}

VA research agendas call for evaluation studies of nonpharmacological therapies for veterans with chronic musculoskeletal pain, including RCTs of dose, frequency, and duration of $\mathrm{CIH}$ treatments, such as chiropractic care $[13,33]$. This qualitative study explored veteran perceptions of the research methods and clinical care received in a pilot trial conducted during the planning phase for a full-scale, multi-site, pragmatic RCT of multimodal chiropractic care [31]. RCTs are a well-established methodology for evaluating chiropractic manipulative therapy [34-40], although few prospective RCTs conducted with veteran populations are reported [15]. Further, few publications describe the lessons learned from the planning or implementation of such intensive research projects, with most papers reporting on the development of credible sham procedures [41-43] or patient recruitment [43-46].

Qualitative interviews conducted with the veterans who enrolled in this pilot trial allowed our team to 
understand potential barriers and facilitators to conducting pragmatic RCTs of chiropractic care in VA settings, findings which may be transferrable to other researchers who plan to conduct clinical trials of chiropractic care and nonpharmacological pain treatments, either within or outside VA healthcare settings. Our pilot study identified critical issues related to treatment scheduling, participant recruitment, and patient communication needs, which we addressed in the development of the full-scale RCT [31]. In addition, these interviews clarified the burden and relevance of selected outcome measures and how best to facilitate online data collection, findings which may help other VA researchers plan their clinical trials, as well as influence how clinicians who deliver manual therapies evaluate patient responses to these treatments. Veterans also offered useful recommendations about the clinic environment within VA and provided insights into the delivery of chiropractic treatments for this patient population, which we describe later in this discussion.

\section{Treatment scheduling}

We sought to understand veterans' experiences of scheduling and attending VA-based chiropractic visits to plan for a multi-site RCT [47]. Scheduling challenges are common barriers to treatment among veterans, including those enrolled in clinical studies $[48,49]$. While most veterans in our pilot reported few scheduling issues, some voiced concerns with wait times for initial visits, limited clinic hours, and a desire for more frequent, or fewer, treatments. Negative perceptions involving VA wait times are well-publicized [50], although a recent study of new patient scheduling for primary care and select specialties reported similar wait times between VA and private sector facilities, with the exception of orthopedic care [51]. In this study, some veterans described "scheduling out" future visits, with cancellations made if a chiropractic appointment was perceived as unneeded. Previous noshows and appointment age (time since appointment scheduled) are predictors of missed appointments in VA [52]. The number of missed appointments for VA chiropractic visits is unknown. However, patient no-shows may account for $18 \%$ of all missed outpatient visits, costing VA upwards of $\$ 167$ per encounter in 2008 [53]. Our team used information about scheduling patterns and concerns to develop a pragmatic treatment protocol and attendance monitoring plan for the full-scale trial.

\section{Outcome measures}

The NIH Task Force on Research Standards for Chronic Low-Back Pain recommends stakeholder assessment of outcomes of most importance to patients [24]. Veterans in this trial considered most selected outcomes relevant for a study of chronic LBP, with some notable comments.
That these veterans approved of personalized questionnaires is consistent with the literature on patient expectations in LBP care and complementary medicine [54-56]. Indeed, recent studies note that patient goals for LBP have only modest alignment with commonly used outcome measures $[56,57]$. Some veterans in this pilot trial reported the outcome measure questionnaires were time-consuming or repetitious to complete. Our team expected such comments as we were piloting newer data collection tools against established chronic pain instruments [26, 27]. Based upon this pilot, future clinical trials of chiropractic care in VA should offer streamlined outcome measures to decrease participant burden [24].

Most veterans (95\%) enrolled in this study had a documented mental health comorbidity, as was consistent with eligibility criteria that did not exclude persons with these diagnoses from participation, which was one reason our protocol included multiple measures of mental health. Some veterans with mental health conditions may be willing to share de-identified data with researchers [58]. However, others remain concerned about the stigma of mental health diagnoses (depression, PTSD) and military sexual trauma and may be reluctant to engage in treatment $[59,60]$. In this trial, veterans often mentioned their specific condition during their interviews. And yet, some negatively viewed research items addressing such topics as alcohol or substance use, anger, and suicide. In addition, the positive outlook subscale of the Healing Encounters and Attitudes List (HEAL), a measure of nonspecific factors in healthcare treatments [61], was considered overtly religious or spiritual by some. Previous research has linked anger with chronic LBP [62] as well as negative spiritual coping or distress with increased mental health diagnoses, symptom severity, and chronic pain [63-65]. Clinical providers, including doctors of chiropractic, should be aware of how common these health concerns are among veterans, institute appropriate assessments, and consider referrals to appropriate specialists for veterans who express emotional or spiritual distress $[19,66,67]$.

\section{Data collection procedures}

While most participants successfully completed online data collection using REDCap via computers or smartphones, almost half of those interviewed expressed at least one challenge in doing so. Veterans report satisfaction and competence with using electronic health information technology (e-health), completing online surveys, and engaging in research using web-based applications [68-70]. However, some veterans report computer literacy challenges or experience difficulty using online resources or electronic health records, including MyHealtheVet [70-73]. Our future trial addresses these 
concerns with additional training on online data collection for participants and tracking protocols for study personnel to assure timely completion of outcome measures.

\section{Participant recruitment}

Our response rates for the overall pilot trial and for this qualitative study (60\%) and the low dropout rates for each met our feasibility goals. This pilot evaluated 3 participant recruitment strategies, including personalized letters sent to veterans who were screened through the electronic health record, focused recruitment from the chiropractic clinic, and provider referrals, along with standard techniques such as study-branded brochures $[74,75]$. Veterans endorsed these strategies, which proved useful in other VA-based studies of non-pharmacological interventions [76]. Our results also echo the reasons why veterans have participated in clinical studies, including valuing altruism by offering their enrollment as service to other veterans [77]. However, our participants did not identify an altruistic desire to "pay back" healthcare professionals as a recruitment motivator, as identified elsewhere [77]. While monetary compensation was not the primary motivator for enrollment, this finding differs from a study of more recent veterans who reported adequate financial compensation plus the opportunity to help other veterans were key considerations for joining VA-based research [78]. Our full-scale trial incorporated this feedback into the study protocol for participant recruitment and retention [31]. Future studies also may consider social media for veteran recruitment into clinical studies, which may be useful for younger veterans, patients engaged in risky behavior, and those who do not currently use VA-located health services [79].

\section{VA-based chiropractic services}

Participants in this small trial offered broad suggestions for VA-based chiropractic services, which our team used in concert with recent trend analyses [17] to create staffing models that meet patient preferences and protocol parameters for the full-scale RCT [31]. While not all of the concerns mentioned by VA patients can be addressed within such a trial (such as room sizes or provider availability), some suggestions might offer insights for longterm planning of VA chiropractic service delivery beyond this single location and research study $[17,80]$.

In 2016, onsite VA clinics served over 37,000 unique chiropractic patients and provided nearly 160,000 chiropractic visits [17]. In this qualitative study, veterans recommended additional chiropractic staff to enhance appointment scheduling, which may be challenging as nearly $25 \%$ of veterans lack adequate access to health professionals, particularly veterans living in designated Shortage County Areas [81]. Key VA stakeholders have made similar observations about the availability of $\mathrm{CIH}$ providers, including chiropractors [82-84]. Improving access to nonpharmacological pain treatments is important, as limited access hampered pain management and increased costs in patients with chronic musculoskeletal pain, especially among younger veterans [85]. Gendersensitive care, including staffing, is advised by women's health experts in VA $[28,86]$. In this pilot study of chiropractic care, a manually delivered treatment, some participants stated a preference to receive care from a female chiropractor, which currently comprise about $20 \%$ of VA chiropractors [17]. Male and female veterans who have experienced military sexual trauma want to choose the gender of their healthcare providers, while many women veterans prefer access to gender distinct clinics and waiting areas [48, 87-89].

Patient preferences for chiropractic clinic environments are not well understood. Key stakeholder groups, especially patients and families, advocate for chiropractic clinics that emphasize comfort, allow for privacy and dignity, and offer 'healing environments' [90-95]. Veterans in this pilot described hospital-like environments that might better attend to lighting levels and ambient noise to enhance patient comfort. VA patients also value clinic settings perceived as safe and private [86]. Our participants also requested information about what to expect from a chiropractic treatment, such as the equipment used, anticipated sounds and sensations, and body positioning [19]. Patient education about practices, procedures, and equipment is a commonly reported unmet need among non-users of chiropractic care [96].

\section{Methodological rigor and study limitations}

Methodological rigor was enhanced through the following strategies [97]. The structured interview guide, interviewer training protocols, peer debriefing with completed transcripts, and previous experience conducting research interviews with patients supported the credibility of these results. Prolonged engagement was established through conducting interviews with multiple participants over the course of the clinical trial. Dependability of findings included the detailed audit trail of coding decisions and extensive use of representative quotes linked to coding themes. Transferability to other VA contexts included data saturation of primary themes across participants, with subthemes included in supplemental tables to identify potential concerns for institutions newly implementing chiropractic care in their settings.

This study has limitations. We did not interview all participants due to scheduling challenges and interviewee non-participation. Those who did not participate, particularly those who self-selected out, may have offered additional perspectives. Veterans who receive 
chiropractic care in other VA facilities or outside the context of a research study might have other opinions. Data were collected in-person and through telephone interviews, and by study coordinators who were either known or unknown to participants. These procedural differences may have influenced the rapport between participant and interviewer and could have impacted data quality or content. Finally, veterans were not compensated for completing the interview, as they were for quantitative measures, which may have impacted participation. Our team has added financial compensation for participants who complete qualitative interviews in the full-scale trial [31].

\section{Conclusions}

This qualitative study highlighted veteran stakeholders' perceptions of the feasibility and acceptability of VAbased chiropractic services for the treatment of chronic LBP. Veterans offered important suggestions for conducting a full-scale, pragmatic randomized controlled trial of multimodal chiropractic care in this clinical setting. Key aspects of clinical trial planning addressed through these interviews included defining treatment scheduling protocols, confirming usefulness of multiple recruitment strategies, refining and streamlining outcome measures, enhancing online data collection procedures, and developing multiple means for communication with participants. Veterans also offered suggestions, such as chiropractic staffing considerations, more clinic-like environments, enhanced patient education, including about the availability of chiropractic services in VA, and patient-centered treatment visits which may be useful in administrative decisions about VA-based chiropractic care.

\footnotetext{
Abbreviations

$\mathrm{ClH}$ : Complementary and integrative health; COCOV: Care Outcomes for Chiropractic Outpatient Veterans (NCT03254719); COREQ: Consolidated Criteria for Reporting Qualitative Studies; DC: Doctor of chiropractic; DSMC: Data and Safety Monitoring Committee; HEAL: Healing Encounters and Attitudes List; IRB: Institutional review board; LBP: Low back pain; MHV: My HealtheVet; MST: Military sexual trauma; NCCIH: National Center for Complementary and Integrative Health; NCRR: National Center for Research Resources; NIH: National Institutes of Health; PASTOR: Pain Assessment Screening Tool and Outcomes Registry; PCP: Primary care provider; PHR: Personal health record; PROMIS ${ }^{\circledR}$ : Patient-Reported Outcome Measurements Information System; PTID: Participant identification number; PTSD: Post-traumatic stress disorder; RCT: Randomized controlled trial; REDCap: Research Electronic Data Capture; SD: Standard deviation; US/USA: United States of America; VA: U.S. Department of Veterans Affairs, Veterans Health Administration; VERDICT:Veteran Response to Dosage in Chiropractic Therapy (NCT04087291); VISN: Veterans Integrated Service Network (US).
}

\section{Supplementary Information}

The online version contains supplementary material available at https://doi. org/10.1186/s40814-021-00962-5.
Additional file 1. Consolidated Criteria for Reporting Qualitative Studies (COREQ) Checklist

Additional file 2. Qualitative Data Tables

\section{Acknowledgements}

The authors sincerely thank all the veterans who participated in the COCOV study. We recognize the contributions of trial co-investigators Dr. Thad Abrams (Site PI), Dr. Anthony Lisi, Dr. Lucille Burgo-Black, Dr. Richard Branson, and those of study personnel including Dr. Julie Hartman, Dr. Janice Hubbard, Tracy Szabo, and Lance Corber. We also appreciate the contributions of Dr. Gina Bonavito-Larragoite and Dr. Gregory Norton who served as the doctors of chiropractic in this study.

\section{Authors' contributions}

CMG, SAS, CRL, and RBW were involved in obtaining funding. The corresponding author, SAS, contributed to all aspects of this paper, including primary responsibilities for developing the qualitative study design and interview schedule, training and oversight of interviewers, data analysis, and manuscript preparation. CMG, RDV, and RBW contributed to the study design, interview questions, and provided critical review of the manuscript. CRL and CMG as CoPls provided oversight of the pilot trial and provided critical feedback on this paper. ET conducted interviews and contributed critical review of the analysis. All authors read and approved the final manuscript.

\section{Funding}

The Collaborative Care for Veterans with Spine Pain and Mental Health Conditions study was funded by Grant R34AT008427 from the National Institutes of Health (NIH), National Center for Complementary and Integrative Health $(\mathrm{NCCIH})$. This study was conducted in a facility constructed with support from a Research Facilities Improvement Program Grant (C06-RR15433) from the National Institutes of Health, National Center for Research Resources (NCRR). The funders had no role in study design, data collection and analysis, decision to publish, or preparation of the manuscript. The contents of this manuscript are solely the responsibility of the authors and do not necessarily represent the official views of $\mathrm{NIH}, \mathrm{NCCIH}$, or the U.S. Department of Veterans Affairs.

\section{Availability of data and materials}

For information about the dataset used in this study, please contact the Office of Data Management and Biostatistics, Palmer Center for Chiropractic Research, Palmer College of Chiropractic, Davenport, lowa at palmer-resea rch@palmer.edu.

\section{Declarations}

\section{Ethics approval and consent to participate}

This research was performed in accordance with the Declaration of Helsinki for Ethical Principles for Medical Research Involving Human Subjects. The study protocol underwent full external peer review from a scientific panel at the National Center for Complementary and Integrative Health $(\mathrm{NCCIH})$ as part of funding, as well as additional protocol reviews by NCCIH and an independent Data and Safety Monitoring Committee (DSMC) before trial launch. Ethical approval for the COCOV Clinical Trial was granted by the Palmer College of Chiropractic IRB (2017G180, May 25, 2017), The University of lowa IRB-03 VA Only (201706802, June 22, 2017), and VA Connecticut Health System IRB (02193, February 1, 2018). All participants provided written informed consent to participate in the clinical trial, including the qualitative interview. Participants additional provided verbal consent for the audiorecording process before the interview. VA patients also signed a document, the Department of Veterans Affairs Authorization for Use and Release of Individually Identifiable Health Information Collected for VHA Research (VA Form 10-0493, Sept. 2015), to permit the use of the audiorecorded interviews for research purposes.

\section{Consent for publication}

Not applicable.

\section{Competing interests}

Stacie A. Salsbury, Elissa Twist, Robert B. Wallace, Robert D. Vining, Christine M. Goertz, and Cynthia R. Long have no competing interests. 


\section{Author details}

'Palmer Center for Chiropractic Research, Palmer College of Chiropractic, 741 Brady Street, Davenport, lowa 52803, USA. ²Department of Epidemiology, College of Public Health, The University of lowa, S422 CPHB, 145 N. Riverside Drive, lowa City, lowa 52242, USA. ${ }^{3}$ Department of Orthopaedic Surgery, Duke University School of Medicine, 200 Morris Street, Durham, North Carolina 27701, USA.

Received: 21 December 2020 Accepted: 13 December 2021 Published online: 14 January 2022

\section{References}

1. Goulet JL, Kerns RD, Bair M, Becker WC, Brennan P, Burgess DJ, et al. The musculoskeletal diagnosis cohort: examining pain and pain care among veterans. Pain. 2016;157(8):1696-703.

2. Gironda RJ, Clark ME, Massengale JP, Walker RL. Pain among veterans of Operations Enduring Freedom and Iraqi Freedom. Pain Med. 2006;7(4):339-43.

3. Haskell SG, Ning Y, Krebs E, Goulet J, Mattocks K, Kerns R, et al. Prevalence of painful musculoskeletal conditions in female and male veterans in 7 years after return from deployment in Operation Enduring Freedom/Operation Iraqi Freedom. Clin J Pain. 2012;28(2):163-7.

4. Higgins DM, Fenton BT, Driscoll MA, Heapy AA, Kerns RD, Bair MJ, et al. Gender differences in demographic and clinical correlates among veterans with musculoskeletal disorders. Womens Health Issues. 2017;27(4):463-70.

5. Outcalt SD, Yu Z, Hoen HM, Pennington TM, Krebs EE. Health care utilization among veterans with pain and posttraumatic stress symptoms. Pain Med. 2014;15(11):1872-9.

6. Beehler GP, Rodrigues AE, Mercurio-Riley D, Dunn AS. Primary care utilization among veterans with chronic musculoskeletal pain: a retrospective chart review. Pain Med. 2013;14(7):1021-31.

7. Gellad WF, Good CB, Shulkin DJ. Addressing the opioid epidemic in the United States: lessons from the Department of Veterans Affairs. JAMA Intern Med. 2017;177(5):611-2.

8. Kligler B, Bair MJ, Banerjea R, DeBar L, Ezeji-Okoye S, Lisi A, et al. Clinical policy recommendations from the VHA State-of-the-Art Conference on Non-Pharmacological Approaches to Chronic Musculoskeletal Pain. J Gen Intern Med. 2018;33(1):16-23.

9. Frank JW, Carey E, Nolan C, Kerns RD, Sandbrink F, Gallagher R, et al. Increased nonopioid chronic pain treatment in the Veterans Health Administration, 2010-2016. Pain Med. 2018;20(5):869-77.

10. Edmond SN, Becker WC, Driscoll MA, Decker SE, Higgins DM, Mattocks $\mathrm{KM}$, et al. Use of non-pharmacological pain treatment modalities among veterans with chronic pain: results from a cross-sectional survey. J Gen Intern Med. 2018:33(1):54-60.

11. Donaldson MT, Polusny MA, MacLehose RF, Goldsmith ES, Hagel Campbell EM, Miron LR, et al. Patterns of conventional and complementary non-pharmacological health practice use by US military veterans: a cross-sectional latent class analysis. BMC Complement Altern Med. 2018;18(1):246

12. Evans EA, Herman PM, Washington DL, Lorenz KA, Yuan A, Upchurch DM, et al. Gender differences in use of complementary and integrative health by U.S. military veterans with chronic musculoskeletal pain. Womens Health Issues. 2018;28(5):379-86.

13. Becker WC, DeBar LL, Heapy AA, Higgins D, Krein SL, Lisi A, et al. A research agenda for advancing non-pharmacological management of chronic musculoskeletal pain: findings from a VHA State-of-the-Art Conference. J Gen Intern Med. 2018;33(1):11-5.

14. Lisi AJ, Corcoran KL, DeRycke EC, Bastian LA, Becker WC, Edmond SN, et al. Opioid use among veterans of recent wars receiving veterans affairs chiropractic care. Pain Med. 2018;19(suppl_1):S54-60.

15. Dougherty PE, Karuza J, Dunn AS, Savino D, Katz P. Spinal manipulative therapy for chronic lower back pain in older veterans: a prospective, randomized, placebo-controlled trial. Geriatr Orthop Surg Rehabil. 2014:5(4):154-64

16. Dougherty PE, Karuza J, Savino D, Katz P. Evaluation of a modified clinical prediction rule for use with spinal manipulative therapy in patients with chronic low back pain: a randomized clinical trial. Chiropr Man Ther. 2014;22(1):41.

17. Lisi AJ, Brandt CA. Trends in the use and characteristics of chiropractic services in the Department of Veterans Affairs. J Manip Physiol Ther. 2016:39(5):381-6.

18. Green BN, Johnson CD, Daniels CJ, Napuli JG, Gliedt JA, Paris DJ. Integration of chiropractic services in military and veteran health care facilities: a systematic review of the literature. J Evid Based Complement Altern Med. 2016:21(2):115-30.

19. Lisi AJ, Salsbury SA, Hawk C, Vining RD, Wallace RB, Branson R, et al. Chiropractic integrated care pathway for low back pain in veterans: results of a Delphi consensus process. J Manip Physiol Ther. 2018;41(2):137-48.

20. O'Cathain A, Thomas KJ, Drabble SJ, Rudolph A, Hewison J. What can qualitative research do for randomised controlled trials? A systematic mapping review. BMJ Open. 2013;3:e002889.

21. Bartlam B, Waterfield J, Bishop A, Holden MA, Barlas P, Ismail KM, et al. The role of qualitative research in clinical trial development: the EASE back study. J Mixed Methods Res. 2018;12(3):325-43.

22. Tong A, Sainsbury P, Craig J. Consolidated criteria for reporting qualitative research (COREQ): a 32-item checklist for interviews and focus groups. Int J Qual Health Care. 2007;19(6):349-57.

23. Harris PA, Taylor R, Thielke R, Payne J, Gonzalez N, Conde JG. Research electronic data capture (REDCap) — a metadata-driven methodology and workflow process for providing translational research informatics support. J Biomed Inform. 2009;42(2):377-81.

24. Deyo RA, Dworkin SF, Amtmann D, Andersson G, Borenstein D, Carragee $E_{c}$ et al. Report of the National Institutes of Health task force on research standards for chronic low back pain. J Manip Physiol Ther. 2014:37(7):449-67.

25. Roland M, Morris R. A study of the natural history of back pain. Part I: Development of a reliable and sensitive measure of disability in low-back pain. Spine. 1983;8(0362-2436):141-4.

26. Broderick JE, DeWitt EM, Rothrock N, Crane PK, Forrest CB. Advances in patient-reported outcomes: the $\mathrm{NIH} \mathrm{PROMIS}{ }^{\circledR}$ measures. EGEMS. 2013;1(1):1015

27. Cook KF, Kallen MA, Buckenmaier C, Flynn DM, Hanling SR, Collins TS, et al. Evaluation of the validity and response burden of patient self-report measures of the Pain Assessment Screening Tool and Outcomes Registry (PASTOR). Mil Med. 2017;182(7):e1851-61.

28. Yano EM, Hayes P, Wright S, Schnurr PP, Lipson L, Bean-Mayberry B, et al. Integration of women veterans into VA quality improvement research efforts: what researchers need to know. J Gen Intern Med. 2010;25(1):56-61

29. Verhoef MJ, Casebeer AL, Hilsden RJ. Assessing efficacy of complementary medicine: adding qualitative research methods to the "gold standard". J Altern Complement Med. 2002;8(3):275-81.

30. Eldridge SM, Chan CL, Campbell MJ, Bond CM, Hopewell S, Thabane L, et al. CONSORT 2010 statement: extension to randomised pilot and feasibility trials. BMJ. 2016;355:15239.

31. Long CR, Lisi AJ, Vining RD, Wallace RB, Salsbury SA, Shannon ZK, et al. Veteran Response to Dosage in Chiropractic Therapy (VERDICT): study protocol of a pragmatic randomized trial for chronic low back pain. Pain Med. 2020:21(Supplement 2):S37-44.

32. Hsieh HF, Shannon SE. Three approaches to qualitative content analysis. Qual Health Res. 2005;15(9):1277-88.

33. Han L, Goulet JL, Skanderson M, Bathulapalli H, Luther SL, Kerns RD, et al. Evaluation of complementary and integrative health approaches among US veterans with musculoskeletal pain using propensity score methods. Pain Med. 2019;20(1):90-102.

34. Eklund A, Jensen I, Lohela-Karlsson M, Hagberg J, Leboeuf-Yde C, Kongsted A, et al. The Nordic Maintenance Care program: effectiveness of chiropractic maintenance care versus symptom-guided treatment for recurrent and persistent low back pain-A pragmatic randomized controlled trial. PLoS One. 2018:13(9):e0203029.

35. Eklund A, Hagberg J, Jensen I, Leboeuf-Yde C, Kongsted A, Lövgren P, et al. The Nordic maintenance care program: maintenance care reduces the number of days with pain in acute episodes and increases the length of pain free periods for dysfunctional patients with recurrent and persistent low back pain - a secondary analysis of a pragmatic randomized controlled trial. Chiropr. Man Ther. 2020;28(1):19. 
36. Haas M, Vavrek D, Peterson D, Polissar N, Neradilek MB. Dose-response and efficacy of spinal manipulation for care of chronic low back pain: a randomized controlled trial. Spine J. 2014;14(7):1106-16.

37. Karpouzis F, Bonello R, Pribicevic M, Kalamir A, Brown BT. Quality of reporting of randomised controlled trials in chiropractic using the CONSORT checklist. Chiropr Man Ther. 2016;24(1):19.

38. Vining R, Long CR, Minkalis A, Gudavalli MR, Xia T, Walter J, et al. Effects of chiropractic care on strength, balance, and endurance in active-duty U.S. military personnel with low back pain: a randomized controlled trial. J Altern Complement Med. 2020;26(7):592-601.

39. Goertz CM, Long CR, Vining RD, Pohlman KA, Walter J, Coulter I. Effect of usual medical care plus chiropractic care vs usual medical care alone on pain and disability among US service members with low back pain: a comparative effectiveness clinical trial. JAMA Netw Open 2018;1(1):e180105.

40. Goertz CM, Salsbury SA, Long CR, Vining RD, Andresen AA, Hondras MA, et al. Patient-centered professional practice models for managing low back pain in older adults: a pilot randomized controlled trial. BMC Geriatr. 2017;17(1):235.

41. Gudavalli MR, Salsbury SA, Vining RD, Long CR, Corber L, Patwardhan AG, et al. Development of an attention-touch control for manual cervical distraction: a pilot randomized clinical trial for patients with neck pain. Trials. 2015;16:259.

42. Vernon H, MacAdam K, Marshall V, Pion M, Sadowska M. Validation of a sham manipulative procedure for the cervical spine for use in clinical trials. J Manip Physiol Ther. 2005;28(9):662-6.

43. Hawk C, Long CR, Reiter R, Davis CS, Cambron JA, Evans R. Issues in planning a placebo-controlled trial of manual methods: results of a pilot study. J Altern Complement Med. 2002;8(1):21-32.

44. Cambron JA, Dexheimer JM, Chang M, Cramer GD. Recruitment methods and costs for a randomized, placebo-controlled trial of chiropractic care for lumbar spinal stenosis: a single-site pilot study. J Manip Physiol Ther. 2010;33(1):56-61.

45. Cambron JA, Hawk C, Evans R, Long CR. Recruitment and accrual of women in a placebo-controlled clinical pilot study on manual therapy. J Manip Physiol Ther. 2004;27(5):299-305.

46. Hondras MA, Long CR, Haan AG, Spencer LB, Meeker WC. Recruitment and enrollment for the simultaneous conduct of 2 randomized controlled trials for patients with subacute and chronic low back pain at a CAM research center. J Altern Complement Med. 2008;14(8):983-92.

47. Kerns RD, Brandt CA, Peduzzi P. NIH-DoD-VA Pain Management Collaboratory. Pain Med. 2019;20(12):2336-45

48. Martinez ME, Kearney DJ, Simpson T, Felleman BI, Bernardi N, Sayre G. Challenges to enrollment and participation in mindfulness-based stress reduction among veterans: a qualitative study. J Altern Complement Med. 2015:21(7):409-21.

49. Cheney AM, Koenig CJ, Miller CJ, Zamora K, Wright P, Stanley R, et al. Veteran-centered barriers to VA mental healthcare services use. BMC Health Serv Res. 2018;18(1):591.

50. Shulkin D. Understanding veteran wait times. Ann Intern Med. 2017:167(1):52-4

51. Penn M, Bhatnagar S, Kuy S, Lieberman S, Elnahal S, Clancy C, et al. Comparison of wait times for new patients between the private sector and United States Department of Veterans Affairs medical centers. JAMA Netw Open. 2019;2(1):e187096-6.

52. Goffman RM, Harris SL, May JH, Milicevic AS, Monte RJ, Myaskovsky L, et al. Modeling patient no-show history and predicting future outpatient appointment behavior in the Veterans Health Administration. Mil Med. 2017;182(5-6):e1708-14.

53. Kheirkhah P, Feng Q, Travis LM, Tavakoli-Tabasi S, Sharafkhaneh A. Prevalence, predictors and economic consequences of no-shows. BMC Health Serv Res. 2016;16(1):13.

54. Hopayian K, Notley C. A systematic review of low back pain and sciatica patients' expectations and experiences of health care. Spine J. 2014;14(8):1769-80.

55. Foley $\mathrm{H}$, Steel A. Patient perceptions of clinical care in complementary medicine: a systematic review of the consultation experience. Patient Educ Couns. 2017:100(2):212-23.

56. Gardner T, Refshauge K, McAuley J, Goodall S, Hübscher M, Smith L. Patient led goal setting in chronic low back pain-what goals are important to the patient and are they aligned to what we measure? Patient Educ Couns. 2015;98(8):1035-8.

57. Maiers M, Hondras MA, Salsbury SA, Bronfort G, Evans R. What do patients value about spinal manipulation and home exercise for back-related leg pain? A qualitative study within a controlled clinical trial. Man Ther. 2016;26:183-91.

58. Funderburk JS, Spinola S, Maisto SA. Mental health predictors of veterans willingness to consider research participation. Mil Med. 2015;180(6):697-701.

59. DeViva JC, Sheerin CM, Southwick SM, Roy AM, Pietrzak RH, HarpazRotem I. Correlates of VA mental health treatment utilization among OEF/OIF/OND veterans: Resilience, stigma, social support, personality, and beliefs about treatment. Psychol Trauma Theory Res Pract Policy. 2016;8(3):310-8.

60. Botero G, Rivera NI, Calloway SC, Ortiz PL, Edwards E, Chae J, et al. A lifeline in the dark: breaking through the stigma of veteran mental health and treating America's combat veterans. J Clin Psychol. 2020;76(5):831-40

61. Greco CM, Yu L, Johnston KL, Dodds NE, Morone NE, Glick RM, et al. Measuring nonspecific factors in treatment: item banks that assess the healthcare experience and attitudes from the patient's perspective. Qual Life Res. 2016;25(7):1625-34.

62. Burns JW. Arousal of negative emotions and symptom-specific reactivity in chronic low back pain patients. Emotion. 2006;6(2):309-19.

63. Smith-MacDonald L, Norris JM, Raffin-Bouchal S, Sinclair S. Spirituality and mental well-being in combat veterans: a systematic review. Mil Med. 2017;182(11-12):e1920-40.

64. Harris J, Usset T, Krause L, Schill D, Reuer B, Donahue R, et al. Spiritual/ religious distress is associated with pain catastrophizing and interference in veterans with chronic pain. Pain Med. 2018;19(4):757-63.

65. Sharma V, Marin DB, Koenig HK, Feder A, lacoviello BM, Southwick SM, et al. Religion, spirituality, and mental health of U.S. military veterans: results from the National Health and Resilience in Veterans Study. J Affect Disord. 2017;217:197-204

66. Pyne JM, Rabalais A, Sullivan S. Mental health clinician and community clergy collaboration to address moral injury in veterans and the role of the Veterans Affairs chaplain. J Health Care Chaplain. 2019;25(1):1-19.

67. National Academies of Sciences, Engineering, and Medicine; Health and Medicine Division; Board on Health Care Services; Committee, Division $H$ and $M$, Services B on HC, Services $C$ to $E$ the D of VAMH. The Veterans Health Administration's Mental Health Services: Evaluation of the Department of Veterans Affairs Mental Health Services. National Academies Press (US); 2018. [cited 2020 Jul 27]. Available from: https://www.ncbi.nlm. nih.gov/books/NBK499499/

68. Nazi KM, Hogan TP, Mclnnes DK, Woods SS, Graham G. Evaluating patient access to electronic health records: results from a survey of veterans. Med Care. 2013;51(3):S52-6.

69. Whealin JM, Jenchura EC, Wong AC, Zulman DM. How veterans with post-traumatic stress disorder and comorbid health conditions utilize ehealth to manage their health care needs: a mixed-methods analysis. J Med Internet Res. 2016;18(10):e280.

70. Moin T, Ertl K, Schneider J, Vasti E, Makki F, Richardson C, et al. Women veterans' experience with a web-based diabetes prevention program: a qualitative study to inform future practice. J Med Internet Res. 2015;17(5):e127.

71. Turvey C, Klein D, Fix G, Hogan TP, Woods S, Simon SR, et al. Blue Button use by patients to access and share health record information using the Department of Veterans Affairs' online patient portal. J Am Med Inform Assoc. 2014;21(4):657-63.

72. Haun JN, Lind JD, Shimada SL, Martin TL, Gosline RM, Antinori N, et al. Evaluating user experiences of the secure messaging tool on the Veterans Affairs' patient portal system. J Med Internet Res. 2014;16(3):e75.

73. Mishuris RG, Stewart M, Fix GM, Marcello T, Mclnnes DK, Hogan TP, et al. Barriers to patient portal access among veterans receiving home-based primary care: a qualitative study. Health Expect. 2015;18(6):2296-305.

74. Michalek AK, Kan D, Prochaska J. Engaging veterans with substance abuse disorders into a research trial: success with study branding, networking, and presence. Transl Behav Med. 2015;5(2):167-76.

75. Bayley PJ, Kong JY, Helmer DA, Schneiderman A, Roselli LA, Rosse SM, et al. Challenges to be overcome using population-based sampling 
methods to recruit veterans for a study of post-traumatic stress disorder and traumatic brain injury. BMC Med Res Methodol. 2014;14(1):48.

76. Hawkins MS, Hough LJ, Berger MA, Mor MK, Steenkiste AR, Gao S, et al. Recruitment of veterans from primary care into a physical activity randomized controlled trial: the experience of the VA-STRIDE study. Trials. 2014;15(1):11.

77. Campbell HM, Raisch DW, Sather MR, Warren SR, Segal AR. A comparison of veteran and nonveteran motivations and reasons for participating in clinical trials. Mil Med. 2007;172(1):27-30

78. Littman AJ, True G, Ashmore E, Wellens T, Smith NL. How can we get Iraqand Afghanistan-deployed US Veterans to participate in health-related research? Findings from a national focus group study. BMC Med Res Methodol. 2018;18(1):88.

79. Pedersen ER, Helmuth ED, Marshall GN, Schell TL, PunKay M, Kurz J. Using Facebook to recruit young adult veterans: online mental health research. JMIR Res Protoc. 2015;4(2):e63.

80. Lisi AJ, Khorsan R, Smith MM, Mittman BS. Variations in the implementation and characteristics of chiropractic services in VA. Med Care. 2014;52(12 Suppl 5):S97-104.

81. Doyle JM, Streeter RA. Veterans' location in health professional shortage areas: implications for access to care and workforce supply. Health Serv Res. 2017;52(S1):459-80.

82. Fletcher CE, Mitchinson AR, Trumble EL, Hinshaw DB, Dusek JA. Perceptions of other integrative health therapies by veterans with pain who are receiving massage. J Rehabil Res Dev. 2016;53(1):117-26.

83. Fletcher CE, Mitchinson AR, Trumble E, Hinshaw DB, Dusek JA. Providers' and administrators' perceptions of complementary and integrative health practices across the Veterans Health Administration. J Altern Complement Med. 2017;23(1):26-34.

84. Taylor SL, Bolton R, Huynh A, Dvorin K, Elwy AR, Bokhour BG, et al. What should health care systems consider when implementing complementary and integrative health: lessons from Veterans Health Administration. J Altern Complement Med. 2019;25(S1):S52-60.

85. Herman PM, Yuan AH, Cefalu MS, Chu K, Zeng Q, Marshall N, et al. The use of complementary and integrative health approaches for chronic musculoskeletal pain in younger US Veterans: An economic evaluation. PLoS One. 2019;14(6):e0217831.

86. deKleijn M, Lagro-Janssen ALM, Canelo I, Yano EM. Creating a roadmap for delivering gender-sensitive comprehensive care for women veterans. Med Care. 2015;53(4 Suppl 1):S156-64.

87. Mengeling MA, Sadler AG, Torner J, Booth BM. Evolving comprehensive VA women's health care: patient characteristics, needs, and preferences. Womens Health Issues. 2011;21 (4, Supplement):S120-9.

88. Turchik JA, McLean C, Rafie S, Hoyt T, Rosen CS, Kimerling R. Perceived barriers to care and provider gender preferences among veteran men who have experienced military sexual trauma: a qualitative analysis. Psychol Serv. 2013;10(2):213-22.

89. Turchik JA, Bucossi MM, Kimerling R. Perceived barriers to care and gender preferences among veteran women who experienced military sexual trauma: a qualitative analysis. Mil Behav Health. 2014;2(2):180-8.

90. Salsbury SA, Vining RD, Gosselin D, Goertz CM. Be good, communicate, and collaborate: a qualitative analysis of stakeholder perspectives on adding a chiropractor to the multidisciplinary rehabilitation team. Chiropr Man Ther. 2018;26(1):29.

91. Shannon ZK, Salsbury SA, Gosselin D, Vining RD. Stakeholder expectations from the integration of chiropractic care into a rehabilitation setting: a qualitative study. BMC Complement Altern Med. 2018;18(1):316.

92. Salsbury SA, Goertz CM, Twist EJ, Lisi AJ. Integration of doctors of chiropractic into private sector health care facilities in the United States: A descriptive survey. J Manip Physiol Ther. 2018;41(2):149-55.

93. Lisi AJ, Salsbury SA, Twist EJ, Goertz CM. Chiropractic integration into private sector medical facilities: A multi-site qualitative case study. J Altern Complement Med. 2018;24(8):792-800.

94. Hennius BJ. Contemporary chiropractic practice in the UK: a field study of a chiropractor and his patients in a suburban chiropractic clinic. Chiropr Man Ther. 2013;21(1):25.

95. Damaske D, McCrossin P, Santoro F, Alcantara J. The beliefs and attitudes of chiropractors and their patients utilising an open practice environment. Eur J Integr Med. 2016;8(4):438-45.

96. Burke A, Nahin RL, Stussman BJ. Limited health knowledge as a reason for non-use of four common complementary health practices. PLoS ONE.
2015;10(6) [cited 2020 Jul 29] Available from: https://www.ncbi.nlm.nih. gov/pmc/articles/PMC4470691/.

97. Bradshaw C, Atkinson S, Doody O. Employing a qualitative description approach in health care research. Glob Qual Nurs Res. 2017:4:2333393617742282.

\section{Publisher's Note}

Springer Nature remains neutral with regard to jurisdictional claims in published maps and institutional affiliations.
Ready to submit your research? Choose BMC and benefit from:

- fast, convenient online submission

- thorough peer review by experienced researchers in your field

- rapid publication on acceptance

- support for research data, including large and complex data types

- gold Open Access which fosters wider collaboration and increased citations

- maximum visibility for your research: over $100 \mathrm{M}$ website views per year

At BMC, research is always in progress.

Learn more biomedcentral.com/submissions 